\title{
The downstream wake response of marine current energy converters operating in shallow tidal flows
}

\author{
Jack Giles $^{1,2^{*}}$, Luke Myers ${ }^{1}$, AbuBakr Bahaj ${ }^{1}$, Bob Shelmerdine ${ }^{2}$ \\ ${ }^{1}$ University of Southampton, Southampton, UK \\ ${ }^{2}$ IT Power Ltd., Bristol, UK \\ *Corresponding author. Tel: +44 7855037885, E-mail: jack.giles@soton.ac.uk
}

\begin{abstract}
This paper presents findings from an experimental study investigating the downstream wake response from marine current energy convertors operating in various degrees of vertical flow constraint. The paper investigates deep vertically unconstrained sites, mid-depth sites and there is a particular emphasis on shallow tidal stream sites. Shallow tidal resources could be utilised for the deployment of first generation farms. The nature of the downstream wake flow will be a critical factor when determining the farm layout and the wake length is heavily influenced by the flow depth or ratio of rotor diameter to flow depth. A porous actuator disk is used to model the marine current energy convertor and an Acoustic Doppler Velocimeter is used to map the downstream wake. Linear scaling of length ratios suggests mid depth sites of $30-50 \mathrm{~m}$ will produce the shortest wake lengths and for deeper and shallower sites the wake length increases. It is hoped that these relationships between vertical flow constraint and wake length will help with the layout design of tidal stream farms.
\end{abstract}

Keywords: wake, vertical flow constraint, shallow tidal flows, farms.

\section{Nomenclature}

$C_{t}$ thrust coefficient

$U_{\text {def }}$ velocity deficit................................... $m \cdot \mathrm{s}^{-1}$

$U_{w} \quad$ wake velocity........................................ $m \cdot s^{-1}$

$U_{o}$ free-stream velocity at hub height ....... $m \cdot \mathrm{s}^{-1}$

I turbulence intensity.................................\%
$\bar{U} \quad$ Mean velocity of sample ....................... $m \cdot \mathrm{s}^{-1}$

$D$ actuator disk diameter...............................m

$u \quad$ downstream velocity component .......... $m \cdot s^{-1}$

$v \quad$ lateral velocity component ................... $m \cdot \mathrm{s}^{-1}$

$w \quad$ vertical velocity component................. $m \cdot \mathrm{s}^{-1}$

\section{Introduction}

Shallow tidal flows hold a number of advantages for first generation tidal stream farms. Shallow flows, of depths less than $20 \mathrm{~m}$, often have a reduced cross-sectional area suitable for energy extraction compared with deeper channels, but they also have other benefits including close proximity to the shore with many sites situated away from shipping channels. This could make construction and grid connection both easier and more economically feasible. Fig. 1 presents results showing potential sites for device deployments in shallow tidal flows in the UK. The data for bathymetry and mean spring peak velocities was obtained from the BWEA "Marine Energy Resource Atlas" [1] and the layers were manipulated using geographic information system (GIS) software. The highlighted areas show sites with depths between 10$20 \mathrm{~m}$ and spring peak velocities of greater than $1.5 \mathrm{~m} / \mathrm{s}$.

When deploying a farm of Marine Current Energy Converters (MCECs), the nature of the downstream wake flow will be a critical factor when determining the farm layout and packing density. It is known that the wake length is heavily influenced by the flow depth or the degree of vertical flow constraint. This paper presents experimental findings of the flow fields around scale MCEC simulators operating in a circulating flume at varying depths to represent the range of depths present at the many sites suitable for MCEC deployment. Examples of shallow tidal sites include; the Bristol Channel, the Humber Estuary and areas around the Channel Islands (see Fig. 1). Deeper flows exist in the Pentland Firth and in various locations around the West of Scotland. 
Previous work presented by Myers et al. [2] concluded that MCECs operating in shallow fastmoving flow regimes will see a difference in the downstream flow field compared with devices installed in deeper water. It was stated that the effects of sea bed proximity have shown that wake recovery is not as favourable when the flow field is very deep beneath the rotor disk. This is due to reduced shear forces and lack of accelerated flow generated by the close proximity of the sea bed and surface that serve to drive wake dissipation. This paper presents work developed from the previous study [2] to further investigate the effects of vertical flow confinement on the downstream wake development of MCECs. A thorough understanding of wake development is critical for the optimisation of the downstream device spacing in tidal stream farms. Minimising the downstream spacing will enable a higher farm device density and hence higher yields from a specific site.

For a multiple-row MCEC array, longitudinal spacing of devices is expected to be great enough to ensure that downstream devices have an incoming flow regime (and hence power production) that is comparable to devices located upstream. However, at spatially constrained sites this approach to spacing may be tightened in order to increase energy capture per surface area of the site and to reduce electrical connection costs. It is postulated that there may be an optimum device height to flow depth ratio that will lead to the minimisation of downstream wake length. For sites that are deeper or shallower than this optimum depth range, the downstream wake length is expected to increase. Whilst an explanation has been provided for deeper flows [2] it is expected that in a very shallow flow vertical blockage is high and flow acceleration above and below the MCEC will be restricted. Both of these factors are expected to result in reduced mixing between the wake and ambient flow thus increasing wake length.

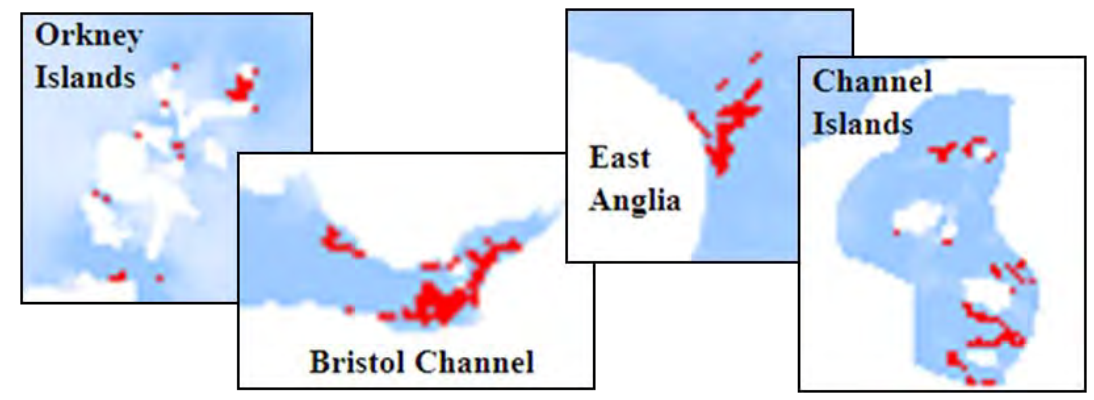

Fig. 1 Potential UK first generation shallow tidal flow sites, not to scale.

\section{Methodology}

In order to conduct the testing at a reasonable scale a porous mesh disk was used to model a horizontal axis turbine (often referred to as actuator disks). Actuators are now an accepted method for modelling MCECs and have been extensively used for horizontal axis turbines, but the method could equally be used to model vertical axis and oscillating hydrofoil devices. Actuator and momentum theory is discussed extensively by Burton et al. [3]. Work concerning the use of small scale actuator disks for the representation of far wake conditions has been addressed by a number of authors for both wind and tidal energy applications [4,5] . The principle difference between flow fields around actuators and full scale MCECs is the representation of the near wake and these differences are generally known to dissipate in less than four rotor diameters downstream [6,7]. 
For this work the principle parameters that require replication from large to small scale are [2]:

a) Device thrust force controlled through the level of actuator disk porosity (ratio of open to closed area).

b) Linear scaling of length ratios such as disk diameter to water depth and channel width.

c) Replication of ambient flow field conditions such as Froude number, vertical velocity profile and turbulence intensities. Full-scale and model Reynolds numbers cannot achieve parity at small scale but should lie within the turbulent classification.

Testing was conducted at a scale of 1:100 using actuator disks of $0.1 \mathrm{~m}$ diameter. The porous actuator's impedance was specified using an empirical relationship between thrust coefficient $\left(\mathrm{C}_{t}\right)$ and plate porosity. This relationship was developed from a combination of experimental findings from the University of Southampton and from equations presented by Whelan et al. [8]. The actuator disk used is of the same porosity as that used in Myers et al. [2].

The actuator disk was mounted on a thin stainless steel support arm which made up part of a pivot arrangement to magnify the small thrust forces on the actuator disk. The rig can be seen in Fig. 2, a 10N button load cell was used to measure the total thrust force.

Shallow-depth experiments were conducted in the tilting flume at the Chilworth hydraulics laboratory, University of Southampton, UK. The working section of this flume is $21 \mathrm{~m}$ in length, $1.37 \mathrm{~m}$ wide and a maximum depth of $0.4 \mathrm{~m}$ for steady operation.

The vertically unconstrained results which were used to compare with the constrained tests were presented by Myers et al. [2] and were conducted in the IFERMER circulating channel, Boulogne sur Mer, France. The channel has a working section of $18 \mathrm{~m}$ in
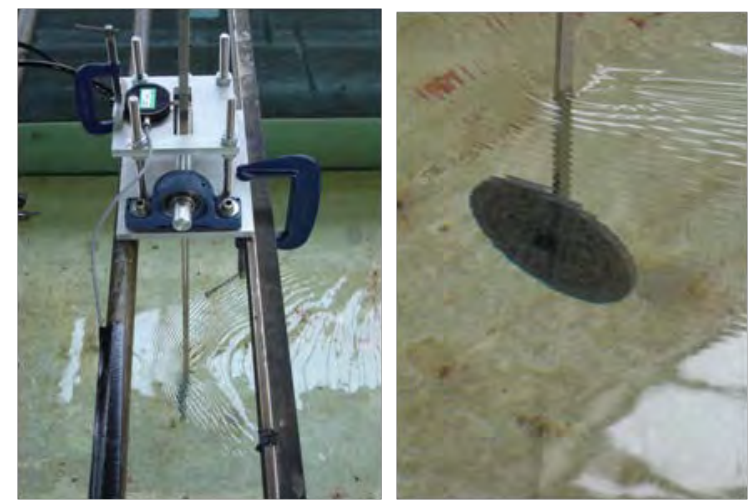

Fig. 2 Actuator lever arm rig (left) actuator disk mounted on lever arm (right)

length, $4 \mathrm{~m}$ wide and $2 \mathrm{~m}$ deep. The downstream wake was mapped using a high frequency Acoustic Doppler Velocimeter (ADV). Operational issues and the accuracy of ADVs have been addressed at length in many publications [9-11]. The ADV was set to sample at $50 \mathrm{~Hz}$. For each data point 7500 readings were taken over a 150 second period.
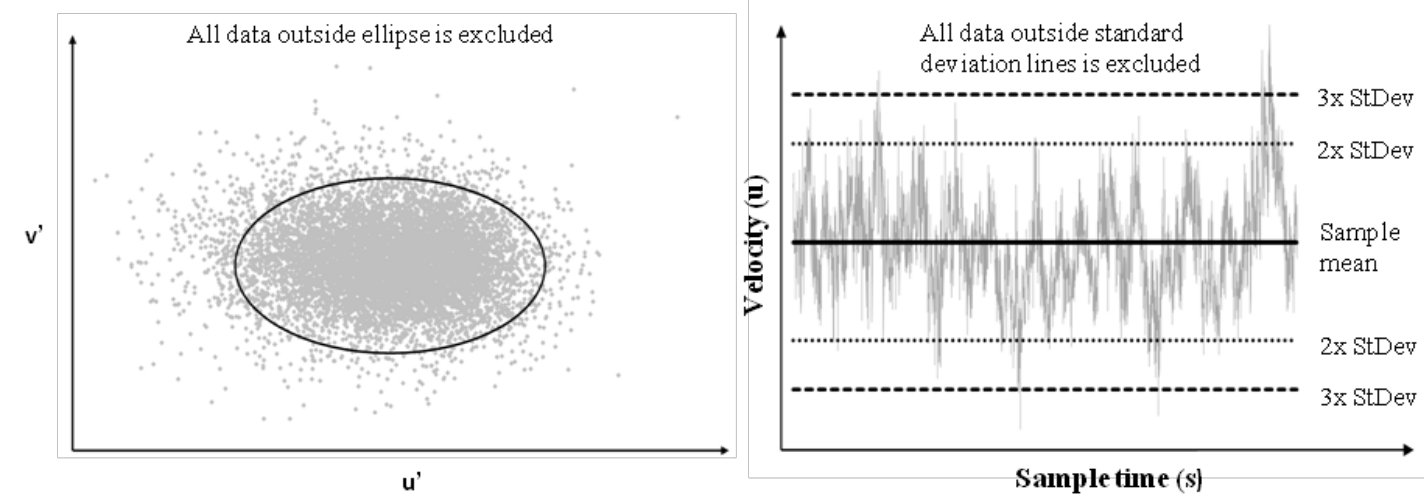

Fig. 3 Velocity correlation filtering method (left) and minimum/maximum filter (right). 
Data was filtered to remove noise and spurious points (Fig. 3, shows data spikes) although the large quantity of suspended particles in the Chilworth channel minimised sample errors. Filtering is required to improve measurements of higher order flow effects such as turbulence intensity and shear stresses as spikes in the data give the impression of increasing energy within the flow. However filtering has a very small effect for mean flow velocities as spikes are generally equally positive and negative. All samples were filtered using a velocity crosscorrelation filter ultimately chosen due to ease of use and effectiveness after a single pass [12]. This method plots the varying components of velocity against each other and constructs an ellipsoid in 3-dimensional space to exclude any data points that deviate significantly from the sample mean (Fig. 3, left). Similar filters can be set up to remove statistically or physically improbable values. Table 1 compares the velocity cross-correlation filter to a minimum-maximum filter (Fig. 3, right) that removes time-series values \pm 3 standard deviations from the sample mean. The effectiveness of the cross-correlation filter for the turbulence data is apparent.

Table 1 Minimum/maximum and velocity correlation filter comparison.

\begin{tabular}{cllllllll}
\hline Sample & \multicolumn{3}{c}{ u-plane velocity $\left(\mathrm{m}^{-1}{ }^{-1}\right)$} & \multicolumn{3}{c}{ u-plane turbulence intensity (\%) } \\
\cline { 2 - 9 } & Raw & $\begin{array}{l}\text { Min/ } \\
\text { max }\end{array}$ & $\begin{array}{l}\text { Vel. } \\
\text { Cor. }\end{array}$ & $\begin{array}{l}\text { \% Change } \\
\text { from raw }\end{array}$ & Raw & $\begin{array}{l}\text { Min/ } \\
\text { max }\end{array}$ & $\begin{array}{l}\text { Vel. } \\
\text { Cor. }\end{array}$ & $\begin{array}{l}\text { \% Change } \\
\text { from raw }\end{array}$ \\
\hline 1 & 0.245 & 0.245 & 0.246 & +0.54 & 9.80 & 9.56 & 7.43 & -24.18 \\
2 & 0.295 & 0.295 & 0.291 & -1.29 & 18.86 & 18.86 & 15.17 & -19.54 \\
3 & 0.286 & 0.286 & 0.286 & -0.11 & 11.30 & 10.59 & 8.91 & -21.14 \\
4 & 0.287 & 0.286 & 0.284 & -0.93 & 13.47 & 11.55 & 10.05 & -25.41 \\
5 & 0.246 & 0.246 & 0.247 & +0.56 & 9.31 & 9.26 & 7.31 & -21.45 \\
\hline
\end{tabular}

The recovery of the wake is defined in terms of velocity deficit; this is a non-dimensional number relative to the free-stream flow speed at hub height and the wake velocity, defined by Eq. (1).

$$
U_{\text {def }}=1-U_{w} / U_{o}
$$

The ambient turbulence intensities in the circulating channel used during this study were approximately $6-8 \%$ and were calculated in all three planes $(\mathrm{u}, \mathrm{v}, \mathrm{w})$. Turbulence intensity is commonly defined as the root-mean-squared of the turbulent velocity fluctuations divided by the mean velocity of the sample. Table 2 details the parameters of the constrained flow tests conducted as part of this work and the previously conducted unconstrained flow tests conducted at the IFERMER facility. Dimensions are detailed in disk diameters (D).

Table 2 experimental test parameters.

\begin{tabular}{cllllll}
\hline Test & $\begin{array}{l}\text { Water } \\
\text { depth }\end{array}$ & $\begin{array}{l}\text { Channel } \\
\text { width }\end{array}$ & $\begin{array}{l}\text { Actuator } \\
\text { centre from } \\
\text { surface }\end{array}$ & $\begin{array}{l}\text { Depth- } \\
\text { averaged } \\
\text { Froude No. }\end{array}$ & $\begin{array}{l}\text { Depth- } \\
\text { averaged } \\
\text { Reynolds No. }\end{array}$ & $\begin{array}{l}\text { Disk } \\
\text { height/depth } \\
\text { ratio }\end{array}$ \\
\hline 1 & 4.0 & 13 & 2.00 & 0.15 & $1.2 \times 10^{5}$ & 0.25 \\
2 & 3.0 & 13 & 1.50 & 0.15 & $7.8 \times 10^{4}$ & 0.33 \\
3 & 2.5 & 13 & 1.25 & 0.15 & $5.7 \times 10^{4}$ & 0.40 \\
4 & 2.0 & 13 & 1.00 & 0.15 & $4.2 \times 10^{4}$ & 0.50 \\
5 & 1.5 & 13 & 0.75 & 0.15 & $2.7 \times 10^{4}$ & 0.66 \\
\hline $6 *$ & 20 & 40 & 2.00 & 0.113 & $9.9 \times 10^{5}$ & 0.05 \\
\hline
\end{tabular}

*unconstrained test conducted at IFERMER, France. 
Myers et al. [2] showed experimentally for a constant depth the wake velocity deficit is independent of velocity (for a representative range of Froude numbers).

\section{Results and Discussion}

Three cases from Table 2 will be addressed herein; A deep-unconstrained tidal site (test \#6), a mid-depth tidal site (test \#1) and a shallow-depth tidal site (test \#4).

\subsection{Free-stream results}

Fig. 4 (left) shows the normalised vertical velocity profiles for the three cases, these are the free-stream results from the Chilworth and IFREMER facilities. Depth is expressed in terms of disk (or rotor, full scale) diameters (D). The velocity profile at Chilworth is well developed but the close proximity of the bed induces a more pronounced gradient that leads to disparate mass flow rates above and below the disk, this is most noticeable in the shallowdepth scenario. Flow speed in the deep site case is similar above and below the disk.

Fig. 4 (right) shows the ambient turbulence intensities in all three planes (u,v,w) for the deepunconstrained and mid-depth scenarios (IFERMER and Chilworth channels, respectively). At the Chilworth facility the presence of the flume bed 2-diameters below the disk causes an increase in turbulence intensity immediately above the bed. $\mathrm{u}$ and $\mathrm{v}$ components are of a similar magnitude at $6-7 \%$ whilst turbulence intensity in the vertical plane is slightly greater. The IFERMER channel turbulence intensity is more constant with depth close to the disk. The turbulence intensity in the vertical plane is much lower than at Chilworth. The difference occurs due to the nature with which water is delivered to the upstream end of the working section.
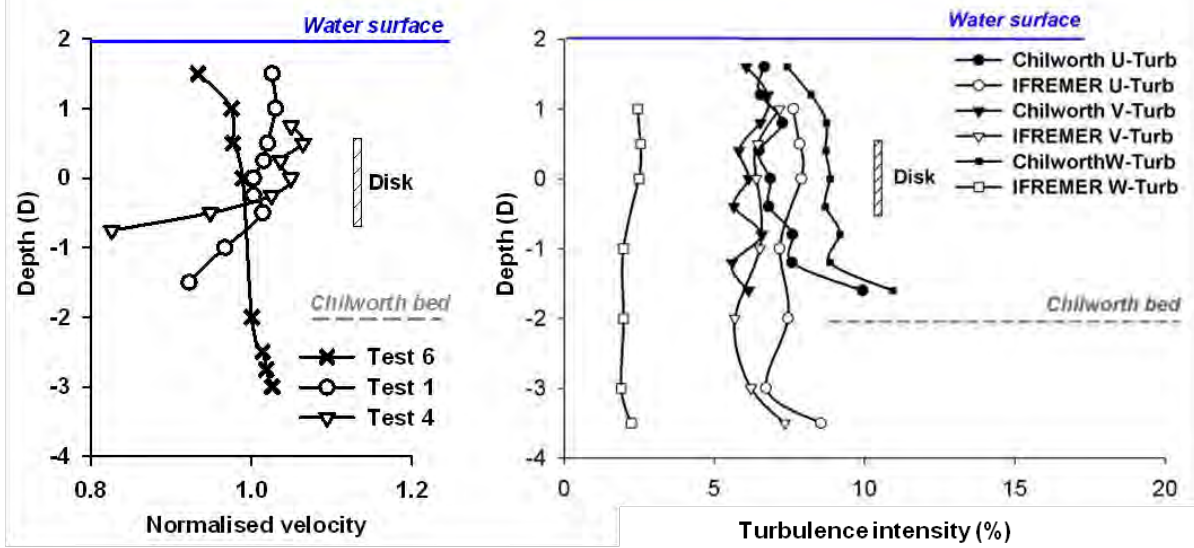

Fig. 4 Normalised vertical velocity profiles at the Chilworth and IFERMER water channels (left) and Turbulence intensities (right).

\subsection{Wake length}

Fig. 5 shows the longitudinal centre plane velocity deficits for the three depth cases. It is clear that in the mid-depth case the wake is broken down in a significantly shorter downstream distance than in the deeper and shallower cases (approximately 6D). This results from flow acceleration above and below the actuator disk that acts to break the wake down through greater lateral turbulent mixing. This effect was postulated by Myers et al. [2] and is reinforced following analysis of these results. 


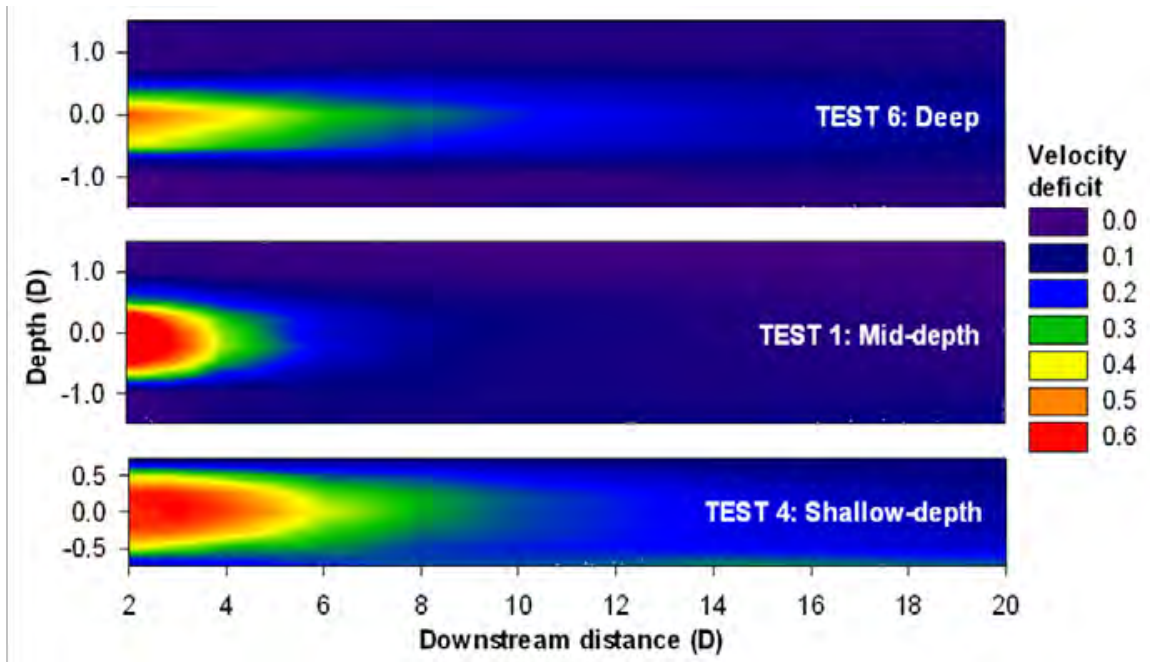

Fig. 5 Centre plane velocity deficit profiles; deep-site, mid-depth site \& shallow-depth site.

The wake persists much further downstream in the deep-unconstrained and shallow depth cases (10-12D downstream), this results from restrictions in flow acceleration around the MCEC. In the deep-unconstrained case vertical blockage is low and hence flow acceleration is reduced, thus allowing the wake to persist further downstream. In the shallow flow scenario vertical blockage is high and hence local flow acceleration above and below the MCEC is restricted, again allowing wake to persist further downstream.
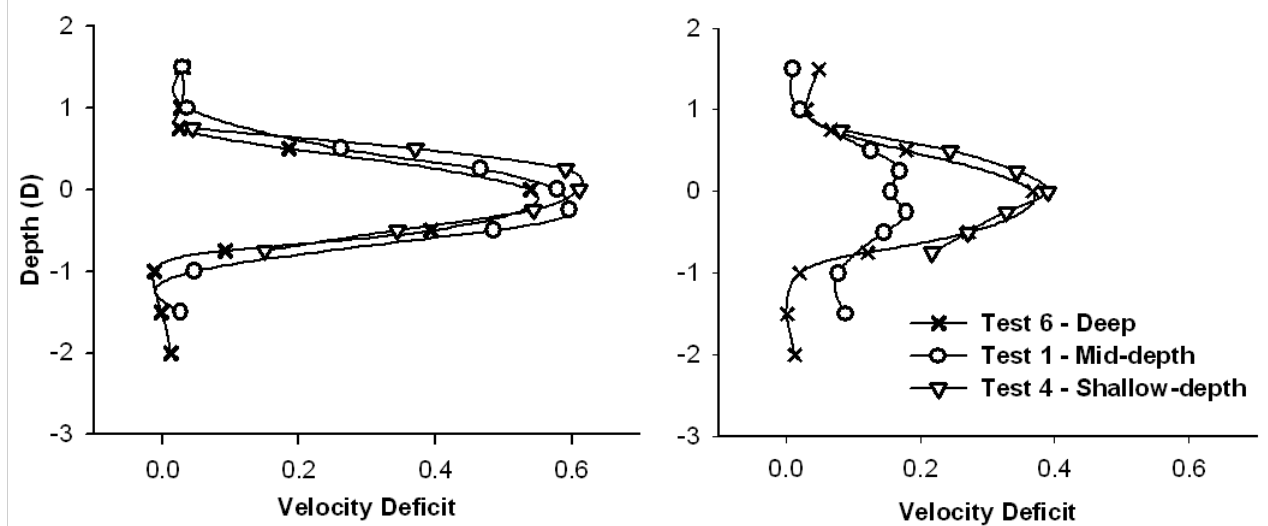

Fig. 6 Vertical velocity deficits at 3 diameters downstream (left) and 6 diameters (right)

Fig. 6 shows vertical line plots of velocity deficit at two downstream locations for all three depth cases. Looking at the 3D downstream graph it is clear that the initial velocity deficits directly behind a MCEC are similar irrespective of the vertical flow constraint; this is because wake is re-energised by turbulent mixing from the surrounding flow and in the near wake this effect is less pronounced. Further downstream e.g. 6D, the effects of varying degrees of vertical blockage can be seen. The deep and shallow cases give similar profiles, whereas the velocity deficits for the mid-depth case are reduced considerably because of increased turbulent mixing between the wake and accelerated surrounding flow. The effects of flow acceleration in the mid-depth case can be observed. 


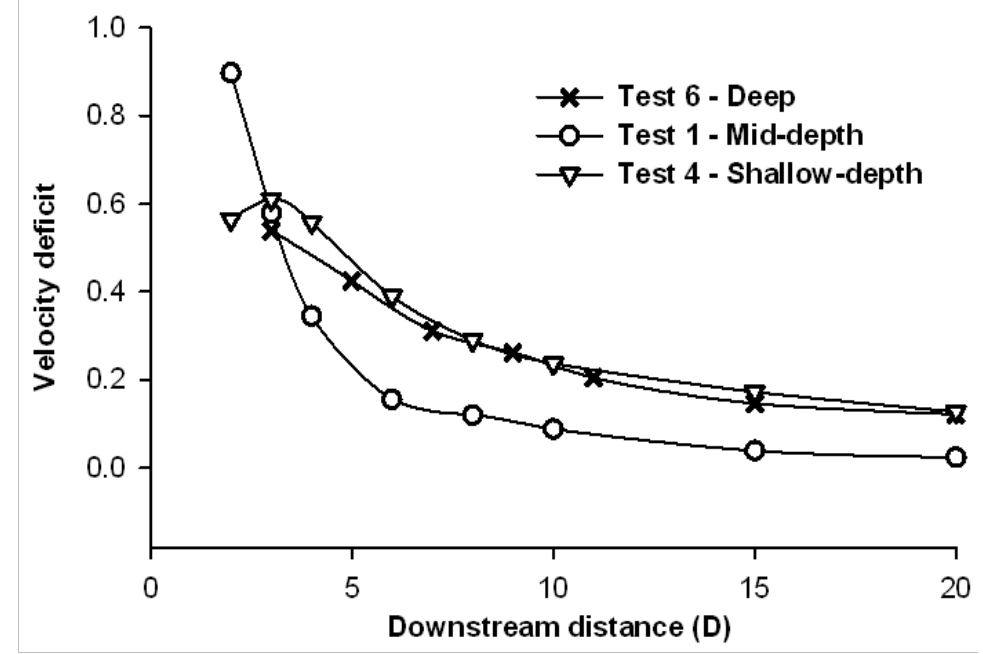

Fig. 7 shows the downstream centreline deficits. As suggested by Fig. 5 the wake recovers much more quickly in the mid-depth case. Again the principal mechanism for this is flow acceleration around the disk which serves to break up the wake more rapidly. In Fig. 7 the similarities in terms of downstream velocity deficits between the shallow and deep cases are clearly illustrated.

Fig. 7 Disk centreline velocity deficit comparison.

\subsection{Farm row optimisation}

This section highlights the significance and importance of this work to tidal stream farm design and optimisation. Fig. 8 shows there is an optimum rotor diameter/flow depth ratio in terms of wake recovery and minimising downstream wake length. Three different downstream location cases are compared for all the tests detailed in Table 2. It appears that 0.25 is the optimum rotor diameter/flow depth ratio for minimising downstream spacing. At full scale this might equate to a site with a depth range of 30-50m depending on the rotor diameter.

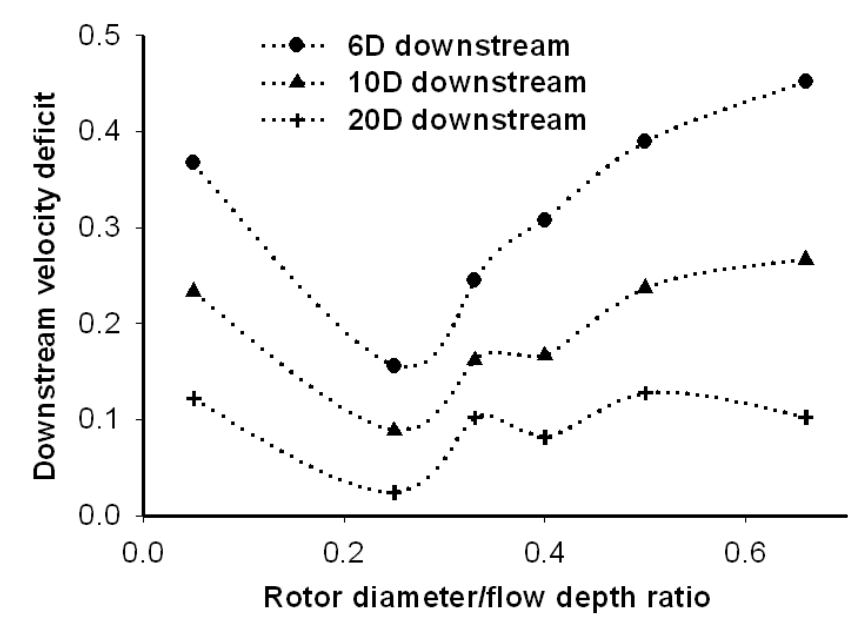

Fig. 8 Optimum rotor diameter/flow depth ratio in terms of wake recovery

\section{Conclusions}

From the results presented in this paper, it is critical that tidal stream farms or arrays are optimised in terms of downstream spacing and packing density, it will thus be important to tune the downstream device spacing to the local flow depth. Although at spatially constrained sites the spacing may be tightened in order to increase energy capture per surface area of the site and to reduce electricity connection costs. It is anticipated that many first generation sites will be located in shallow tidal flows and hence the longer wake lengths compared with middepth sites must be factored into the design process. In terms of the full scale significance and to reduce wake length, the optimum rotor diameter/flow depth ratio is 0.25 . This would equate to a flow depth range of 30-50m depending on the rotor diameter.

The wake length is controlled by the degree of lateral flow mixing between the retarded wake flow and the surrounded accelerated free-stream flow. Increased wake length in very deep and very shallow flows result from vertical blockage, in a deep flow vertical blockage is minimal and hence local flow acceleration around the wake is reduced. In a very shallow flow vertical blockage is high and flow acceleration above and below the MCEC is restricted. Both these 
factors result in less lateral flow mixing and thus increased wake length. It is hoped that the relationship between vertical flow constraint and wake length will help with the layout design of future tidal stream farms.

\section{Acknowledgements}

This work has been conducted as part of an Engineering Doctorate study and has been jointly funded by the European and Physical Sciences Research Council (EPSRC) and IT Power Ltd. Work at IFERMER was funded by the Marine Environment Tests and Research Infrastructure (METRI II) programme and the UK Technology Strategy Board. "Performance characteristics and optimisation of marine current energy converter arrays", project number $\mathrm{T} / 06 / 00241 / 00 / 00$.

\section{References}

[1] ABP, 'Atlas of UK Marine Renewable Energy Resources: Technical Report', ABP Marine Environmental Research Ltd, Department for Business, Enterprise \& Regulatory Reform, 2008.

[2] L. Myers, A.S. Bahaj, G. Germain, J. Giles, 'Flow boundary interaction effects for marine current energy conversion devices', World Renewable Energy Congress X, Glasgow, 2008.

[3] T. Burton, D. Sharpe, N. Jenkins, E. Bossanyi, 'Wind Energy Handbook', Wiley, 2001

[4] A.S. Bahaj, L. Myers, M.D. Thomson, N. Jorge, 'Characterising the wake of horizontal axis marine current turbines’, 7th European Wave and Tidal Energy Conference, Porto, Portugal, 2007.

[5] L. Myers, A.S. Bahaj, R.I. Rawlinson-Smith, M.D. Thomson, 'The effect of boundary proximity upon the wake structure of horizontal axis marine current turbines', 27th International Conference on Offshore Mechanics and Arctic Engineering, Estoril, Portugal, 2008.

[6] P. J. Connel, R.L. George, 'The wake of the MOD-0A1 wind turbine at two rotor diameters downwind on 3 December 1981', Pacific Northwest Laboratory, Battelle, U.S., 1982.

[7] P.E.J. Vermuelen, 'Mixing of simulated wind turbine wakes in turbulent shear flow', TNO Rep. 79-09974, Apeldoorn, Holland, 1979.

[8] J. Whelan, M. Thomson, J.M.R. Graham, J. Peiro, 'Modelling of free surface proximity and wave induced velocities around a horizontal axis tidal stream turbine', 7th European Wave and Tidal Energy Conference, Porto, Portugal, 2007.

[9] A. Lohrmann, R. Cabrera, N. Kraus, M. ASCE, 'Acoustic-Doppler Velocimeter (ADV) for Laboratory Use', Fundamentals and Advancements in Hydraulic Measurements and Experimentation, New York, USA, 1994.

[10]G. Voulgaris, J.H. Trowbridge, 'Evaluation of the Acoustic Doppler Velocimeter (ADV) for Turbulence Measurements', Journal of Atmospheric and Oceanic Technology, 1998, 15, pp. $272-289$.

[11]P.J. Rusello, A. Lohrmann, E. Siegel, T. Maddux, 'Improvements in Acoustic Doppler Velocimetery', The 7th Int. Conf. on Hydroscience and Engineering, Philadelphia, USA, 2006.

[12]L. Cea, J. Puertas, L. Pena, 'Velocity measurements on highly turbulent free surface flow using ADV', Experiments in Fluids, 2007, 42, pp. 333 - 348. 